\title{
IMF effect on sporadic-E layers at two northern polar cap sites: Part I - Statistical study
}

\author{
M. Voiculescu ${ }^{1,2}$, A.T. Aikio ${ }^{2}$, T. Nygrén ${ }^{2}$, and J.M. Ruohoniemi ${ }^{3}$ \\ ${ }^{1}$ Department of Physics, Faculty of Sciences, University "Dunărea de Jos" Galati, 800008 Galati, Romania \\ ${ }^{2}$ Department of Physical Sciences, University of Oulu, P.O. Box 3000, FIN-90014, Finland \\ ${ }^{3}$ Applied Physics Laboratory, The Johns Hopkins University Applied Physics Laboratory, Laurel MD, 20723, USA
}

Received: 10 August 2005 - Revised: 6 February 2006 - Accepted: 13 March 2006 - Published: 19 May 2006

\begin{abstract}
In this paper we investigate the relationship between polar cap sporadic-E layers and the direction of the interplanetary magnetic field (IMF) using a 2-year database from Longyearbyen (75.2 CGM Lat, Svalbard) and Thule (85. $4^{\circ}$ CGM Lat, Greenland). It is found that the MLT distributions of sporadic-E occurrence are different at the two stations, but both are related to the IMF orientation. This relationship, however, changes from the centre of the polar cap to its border. Layers are more frequent during positive $B_{y}$ at both stations. This effect is particularly strong in the central polar cap at Thule, where a weak effect associated with $B_{z}$ is also observed, with positive $B_{z}$ correlating with a higher occurrence of Es. Close to the polar cap boundary, at Longyearbyen, the $B_{y}$ effect is weaker than at Thule. On the other hand, $B_{z}$ plays there an equally important role as $B_{y}$, with negative $B_{z}$ correlating with the Es occurrence. Since Es layers can be created by electric fields at high latitudes, a possible explanation for the observations is that the layers are produced by the polar cap electric field controlled by the IMF. Using electric field estimates calculated by means of the statistical APL convection model from IMF observations, we find that the diurnal distributions of sporadic-E occurrence can generally be explained in terms of the electric field mechanism. However, other factors must be considered to explain why more layers occur during positive than during negative $B_{y}$ and why the $B_{z}$ dependence of layer occurrence in the central polar cap is different from that at the polar cap boundary.
\end{abstract}

Keywords. Ionosphere (Electric fields and currents; Plasma convection; Polar ionosphere)

\section{Introduction}

Sporadic-E (Es) layers are formed by vertical accumulation of ionospheric plasma into thin sheets with high electron density and mainly metallic composition (see, e.g. Whitehead,

Correspondence to: $\mathrm{M}$. Voiculescu

(mirela.voiculescu@ugal.ro)
1970 and references therein; Behnke and Vickrey, 1975). The formation of Es is nowadays generally attributed to the wind shear mechanism (Axford, 1961, 1963; Whitehead, 1961) and/or to directional effect of the electric field (Nygrén et al., 1984; Bristow and Watkins, 1993; Kirkwood and von Zahn, 1991). Appropriate structures in the vertical shear of neutral wind and/or favourable electric field directions create the dynamical conditions for ions to convergence vertically to a certain height. Although at high latitudes both the neutral wind and the electric field may contribute to the formation of sporadic E (e.g. Kirkwood and Nilsson, 2000; Matthews 1998), the electric field often dominates over the neutral wind due to the large values of the electric field (Nygrén, 1990; Parkinson et al., 1998; Bedey and Watkins, 2001).

The plasma convection pattern and the associated electric field at high latitudes and in the polar cap depend strongly on the interplanetary magnetic field (IMF). As a consequence, the Es occurrence is expected to be connected to the IMF direction and strength. Several empirical models of the IMF effect on the convection pattern have been constructed, for example, by Heppner and Maynard (1987), Papitashvili et al. (1994), Ruohoniemi and Greenwald (1996), Weimer (1995), and Papitashvili and Rich (2002). Bristow and Watkins (1994) studied theoretically the effect of different convection patterns on the geographic distribution of Es by applying the Heppner and Maynard (1987) convection model. The IMF-based statistical model of the convection electric field by Weimer (1995) was used by Bedey and Watkins $(1996 ; 1998)$ to explain the observed seasonal and diurnal variations of the Es layers over Sondrestrom. The differences in Es occurrence between the two hemispheres were discussed by Parkinson et al. (1998) on the basis of the IZMEM convection model. Simultaneous IMF and Es observations were compared by Wan et al. (1999) for a southern polar cap station, whereas MacDougall et al. (2000a) studied the Es observations at three northern polar cap sites in the American sector, together with IMF data. Both Wan et al. (1999) and MacDougall et al. (2000a) observed a strong correlation of IMF $B_{y}$ and a weaker correlation of $B_{z}$ with

Published by Copernicus GmbH on behalf of the European Geosciences Union. 


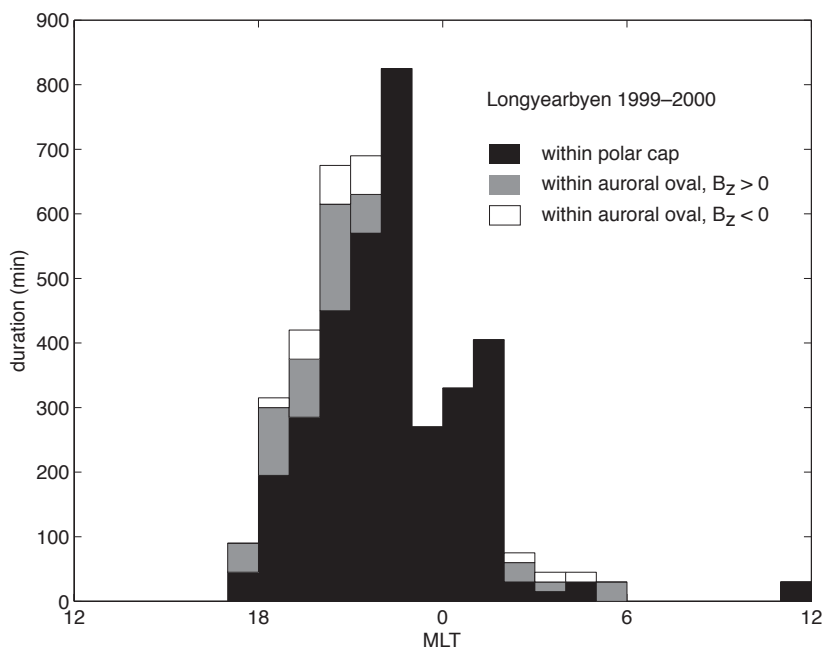

Fig. 1. Total time of Es occurrence at Longyearbyen as a function of MLT at times when the location of the auroral oval was determined by the DMSP satellites. Black columns indicate observations with Longyearbyen inside the polar cap and the other columns indicate those with Longyearbyen in the auroral oval; grey and white during the northward and southward IMF, respectively.

the Es occurrence, regardless of the layer density. However, for a station in the Southern Hemisphere, Wan et al. (1999) found that negative $B_{y}$ correlates with Es occurrence, but for the three stations in the Northern Hemisphere, positive $B_{y}$ was associated with more layers.

Hence, only a few studies with experimental data of polar cap Es and IMF have been conducted so far. The aim of this study is to expand the investigation of the relationship between sporadic $\mathrm{E}$ and the IMF at two high-latitude stations in the Northern Hemisphere, Thule and Longyearbyen, located at different parts of the polar cap. The first one is in the centre, whereas the latter one is located close to the polar cap boundary (for a more detailed discussion about the location of Longyearbyen inside or outside the polar cap, see Sect. 2 of this paper). The database covers two years of observations at both stations and only data fulfilling certain criteria, discussed in Sect. 2, were included. The results of this study are presented in two papers. This paper describes the statistical results of the relationship between the observed IMF orientation and sporadic E occurrence and discusses some possible explanations. The companion paper by Nygrén et al. (2006) (hereafter referred to as Paper II) makes a partial revision of the electric field theory and develops a model probability for Es occurrence at the two sites.

In this study, we will first present the IMF distributions measured by the WIND satellite during the observed sporadic-E layers at the two sites. Electric fields during Es events will next be estimated using IMF observations as input in the statistical APL model (Ruohoniemi and Greenwald, 1996). The relationship between the diurnal Es distributions and the electric fields will then be studied under different
IMF directions. Finally, the observations of this paper together with earlier observations are discussed to find possible explanations for the observed correlations of Es occurrence and IMF direction.

\section{Data analysis}

The two polar cap ionosonde sites chosen for the study are Thule (Greenland, $77.5^{\circ} \mathrm{N}, 290.8^{\circ} \mathrm{E}$ ) and Longyearbyen (Svalbard, $78.2^{\circ} \mathrm{N}, 15.9^{\circ} \mathrm{E}$ ). A database of sporadic-E layers covering two years (1999-2000) from Longyearbyen and two years (1998-1999) from Thule was created. We tried to restrict our study to mid-latitude type layers, which are flat, thin, dense and relatively long-lasting. The parameters relevant to this work are the peak plasma frequency ( $f o E s$ ), and the duration and the local time of occurrence of Es layers. The data coverage was better than $95 \%$ of the total time, so that the results are representative for all seasons and times of day.

In order to exclude unclear cases, we rejected weak layers whose densities were close to the normal E region plasma density, as well as layers with lifetimes shorter than $15 \mathrm{~min}$. Along this line, we selected layers whose peak plasma frequencies were higher than $5 \mathrm{MHz}$ (corresponding to electron densities higher than $3 \cdot 10^{11} \mathrm{~m}^{-3}$ ) and recorded the peak plasma frequency, occurrence time and duration of each event. The Longyearbyen data were available in terms of ionograms at 5-min intervals, whereas only tabulated virtual heights and peak plasma frequencies at 15-min intervals were available from Thule. To obtain the same temporal resolution at both sites, 15-min averages of the peak plasma frequency were calculated for Longyearbyen. Height-spread or auroraltype layers were rejected in Longyearbyen data and, for the same purpose, only layers with the virtual heights within the $90-130 \mathrm{~km}$ range and changing less than $15 \mathrm{~km}$ in $15 \mathrm{~min}$ were accepted in Thule data. The latter choice is based on the observation that the highest velocities of a descending layer are of the order of $20 \mathrm{~km} / \mathrm{h}$ (Kirkwood and Nilsson, 2000), which is equivalent to a $5-10 \mathrm{~km}$ change within the $15-\mathrm{min}$ sampling interval.

The IMF data were supplied by the WIND spacecraft and layer observations from times when WIND was not in the solar wind were rejected (this led to a reduction of about 10\%). Taking into account the time delay of the solar wind from the satellite to the subsolar magnetosphere, the mean values of the three IMF components for each 15-min period of Es occurrence were computed. The ionospheric electric field was then calculated from the observed IMF components $\left(B_{y}\right.$ and $B_{z}$ ) by using the statistical APL convection model (Ruohoniemi and Greenwald, 1996) at the two ionosonde sites. The model is based on ground-based coherent scatter observations of ionospheric convection, as well as patterns derived in satellite studies (Rich and Hairston, 1994; Weimer, 1995). It derives the electrostatic potential within the polar cap as 

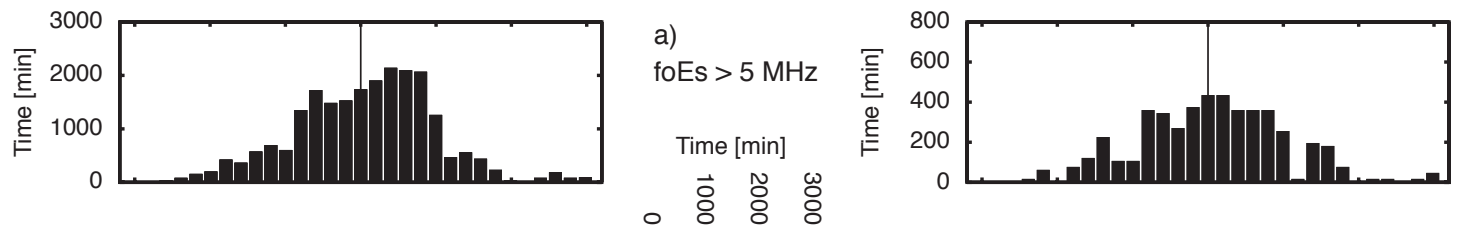

b)
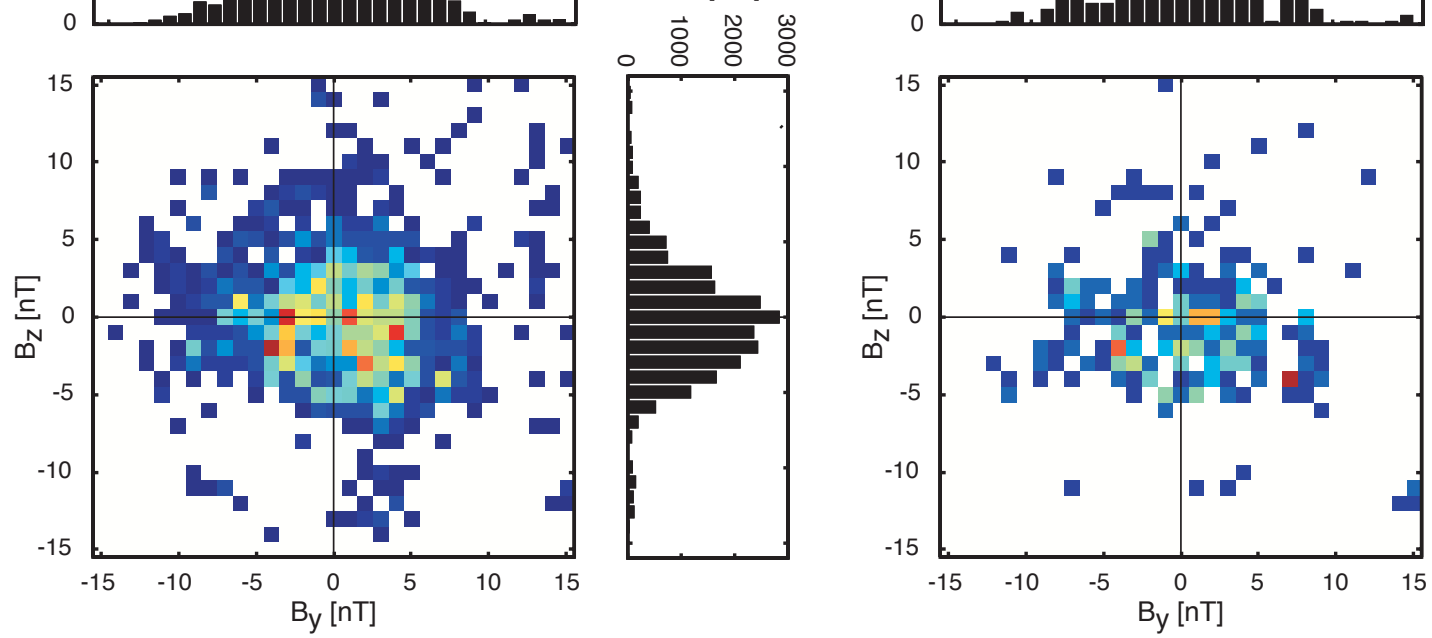

foEs $>10 \mathrm{MHz}$
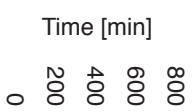

Fig. 2. Bivariate and marginal distributions of sporadic-E occurrence with $B_{y}$ and $B_{z}$ at Longyearbyen in 1999-2000. Distributions (a) and (b) refer to layers with peak plasma densities higher than $5 \mathrm{MHz}$ and $10 \mathrm{MHz}$, respectively. The pixel size in the $B_{y} B_{z}$ plane is $1 \mathrm{nT} \times 1 \mathrm{nT}$. In the colour scale, white indicates zero value, and the colours from blue via yellow and green to red cover the range from small nonzero values to maximum.

series expansions in spherical harmonics for different IMF conditions.

Thule $\left(85.4^{\circ} \mathrm{CGM}\right.$ Lat) is located in the central polar cap, whereas the geomagnetic latitude of Longyearbyen (75.2 $2^{\circ}$ CGM Lat) is about $10^{\circ}$ lower than Thule. At high values of the $\mathrm{Kp}$ index, the Feldstein oval (Feldstein and Starkov, 1967) shows that Longyearbyen is located within the low-latitude region of the polar cap at all other MLT times except 11-13 MLT. During low to moderate Kp values Longyearbyen lies within the polar cap from 18 MLT through midnight to 09 MLT. Hence, Longyearbyen is not necessarily a polar cap site thoroughout the day. In order to check this more specifically, we made an attempt to study the Longyearbyen location with respect to the auroral oval during the observations. For that purpose, we utilised the OVATION web-server provided by JHU/APL (http://sd-www.jhuapl.edu/Aurora/ovation/index.html). We checked all the time intervals of Es observations at Longyearbyen for oval location. OVATION data (provided by the DMSP satellites) were found only for about $18 \%$ of the data points. The result is shown in Fig. 1. Indeed, in most cases $(82 \%)$, Longyearbyen lies within the polar cap (black bars). Sometimes Longyearbyen is located within the auroral oval; typically this happens when IMF points northwards (grey), i.e. when the oval is expected to be contracted. In a few cases this also happens during southward IMF (white); these examples may come from auroral disturbances dur- ing the substorm expansion phase when the oval is very wide and expands to the polar cap. Very few dayside layers were observed at Longyearbyen, and therefore the location of Longyearbyen at daytime is not relevant to this study. In conclusion, Fig. 1 gives a reason to believe that only a small fraction of the layers observed at Longyearbyen come from time intervals when Longyearbyen has not been a polar cap site and therefore the observations are representative of polar cap sporadic E.

\section{IMF and Es layers}

\subsection{Direction of the IMF during observed Es}

Figures 2 and 3 show the bivariate and marginal distributions of sporadic-E occurrence as functions of $B_{y}$ and $B_{z}$ at Longyearbyen and Thule, respectively. Panel (a) shows the distributions for all observed layers (i.e. $f o E s>5 \mathrm{MHz}$ ) and panel (b) only for the most dense layers ( $f o E s>10 \mathrm{MHz}$ ). The bivariate distributions show the projection of the IMF in the $B_{y} B_{z}$ plane during observed Es layers, whereas the marginal distributions show each IMF component separately.

The maxima of the two bivariate distributions at Longyearbyen are close to the origin of the $B_{y} B_{z}$ plane, but the distributions are asymmetric in such a manner that the marginal distributions give more weight to positive $B_{y}$ and negative $B_{z}$. Also, the marginal distributions with $B_{z}$ are more narrow 
Thule 1998-1999
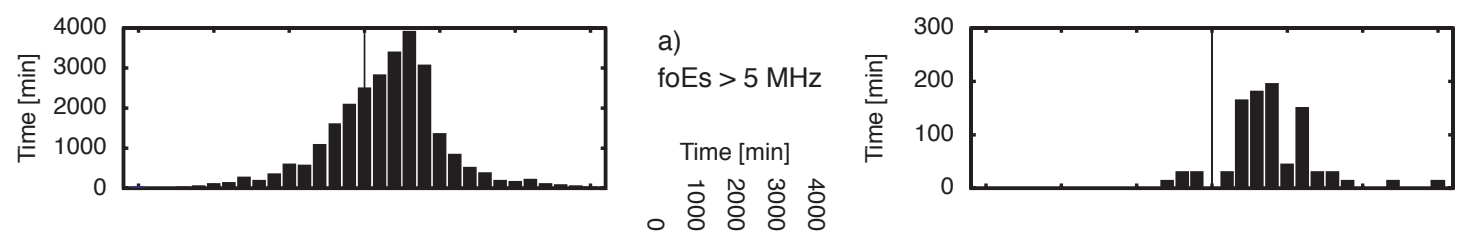

b)
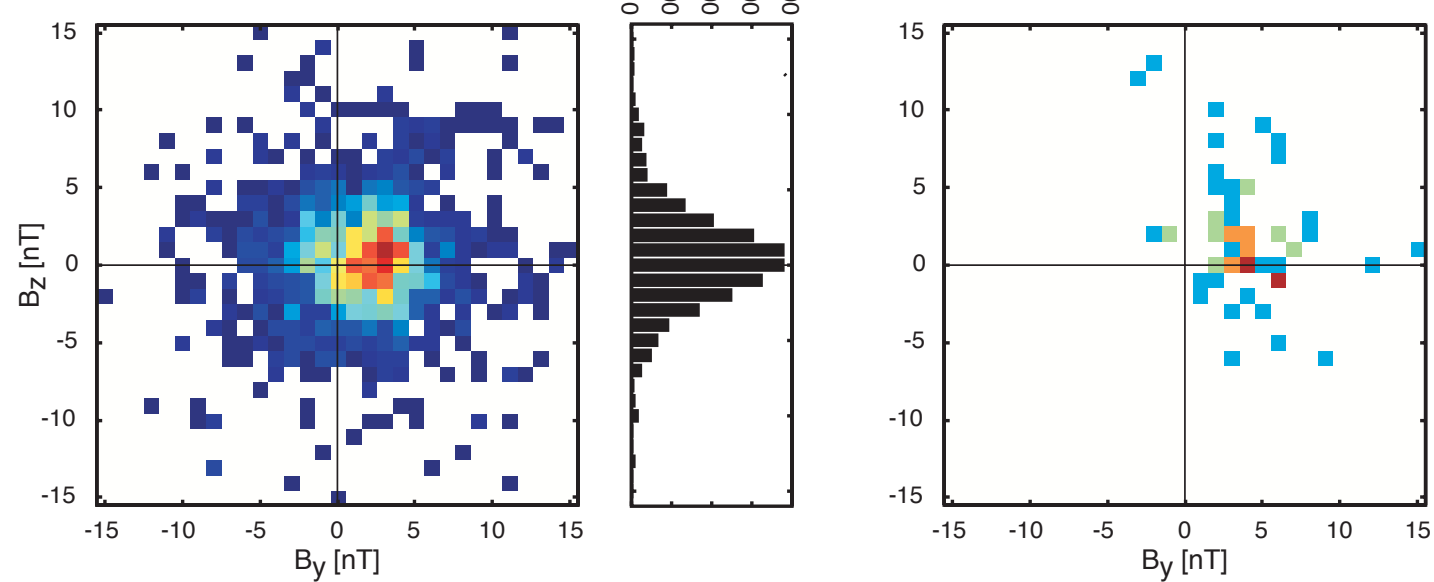

foEs $>10 \mathrm{MHz}$

Time [min]
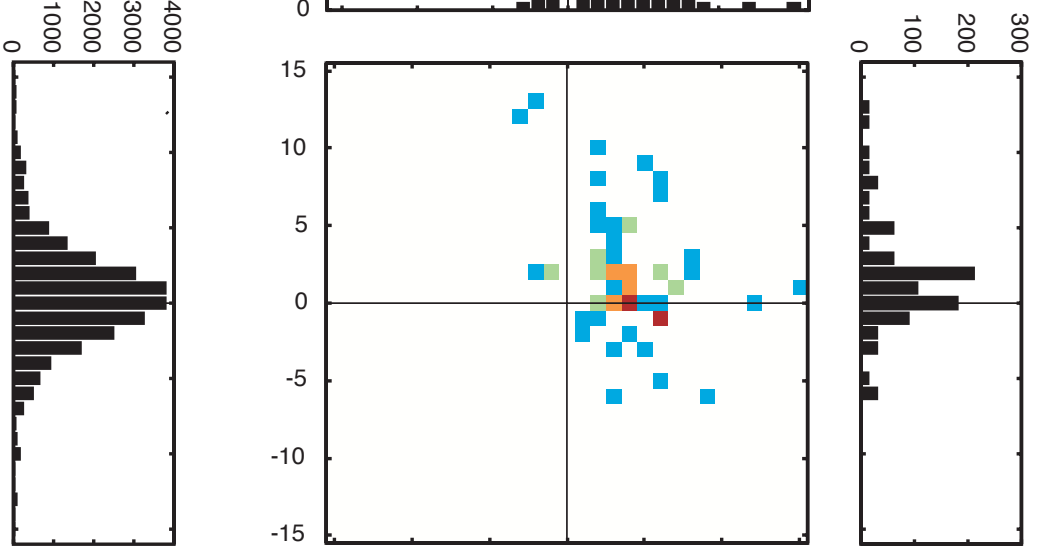

Fig. 3. Same as Fig. 2 for Thule in 1998-1999.

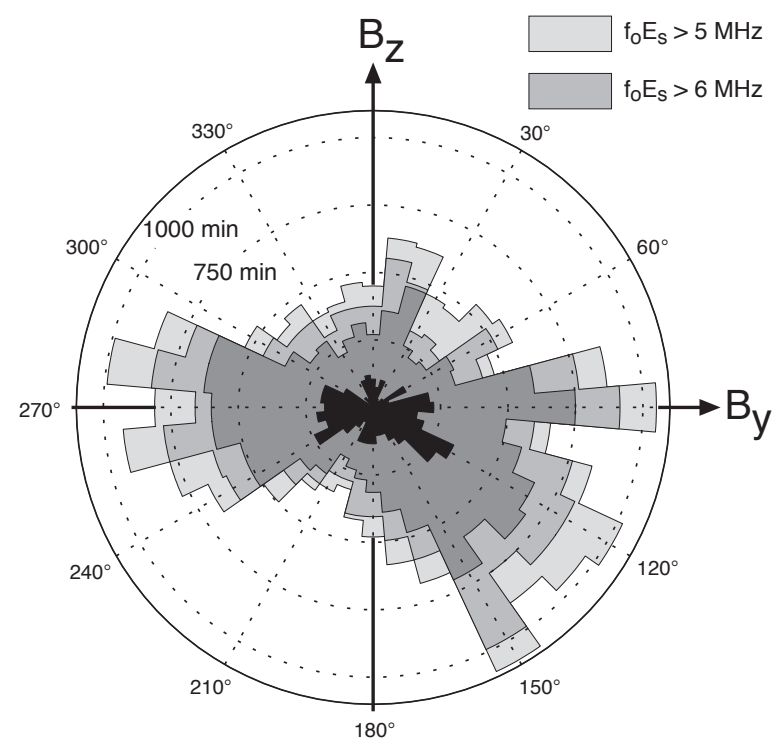

Longyearbyen, 1999-2000

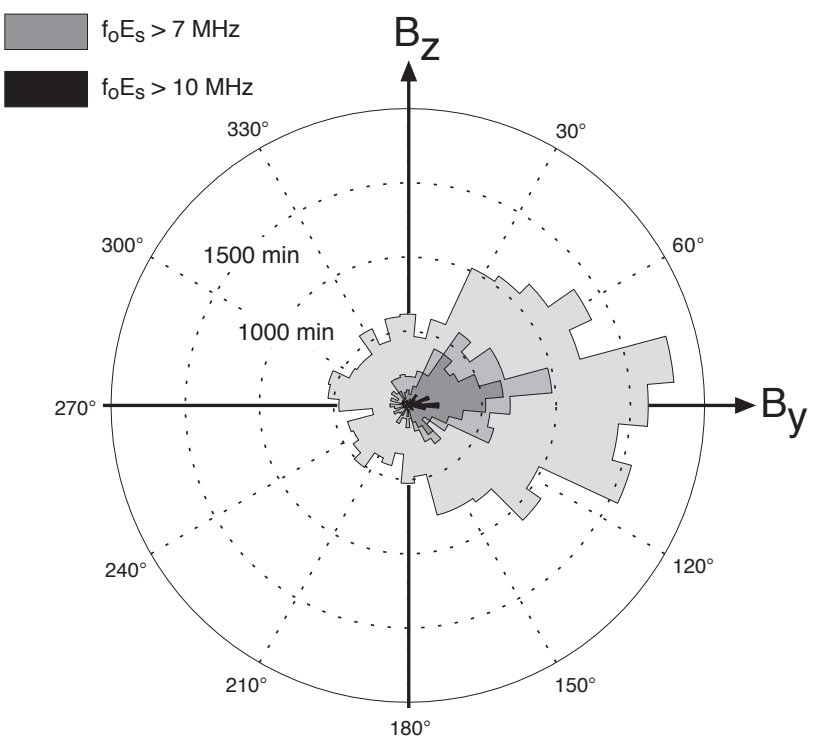

Thule, 1998-1999

Fig. 4. Distributions of Es occurrence at Longyearbyen and Thule as a function of the clock angle of the IMF in the $B_{y} B_{z}$ plane. Separate distributions are plotted for layers with peak plasma frequencies higher than 5, 6, 7 and $10 \mathrm{MHz}$.

than those with $B_{y}$. All this indicates that the Es occurrence is related to the IMF. In the case of the dense layers this is even more obvious since the tendency towards negative $B_{z}$ becomes more apparent.

At Thule the IMF-Es relationship looks different. There the maximum of the bivariate distribution is shifted towards positive $B_{y}$ and slightly towards positive $B_{z}$. This becomes more evident for dense layers, which occur almost exclusively when $B_{y}$ is positive. The same effect is seen in the marginal distributions, as well. For all layers (panel a) the marginal $B_{y}$ distribution is clearly shifted towards positive $B_{y}$, but the marginal $B_{z}$ distribution is nearly symmetric. For 

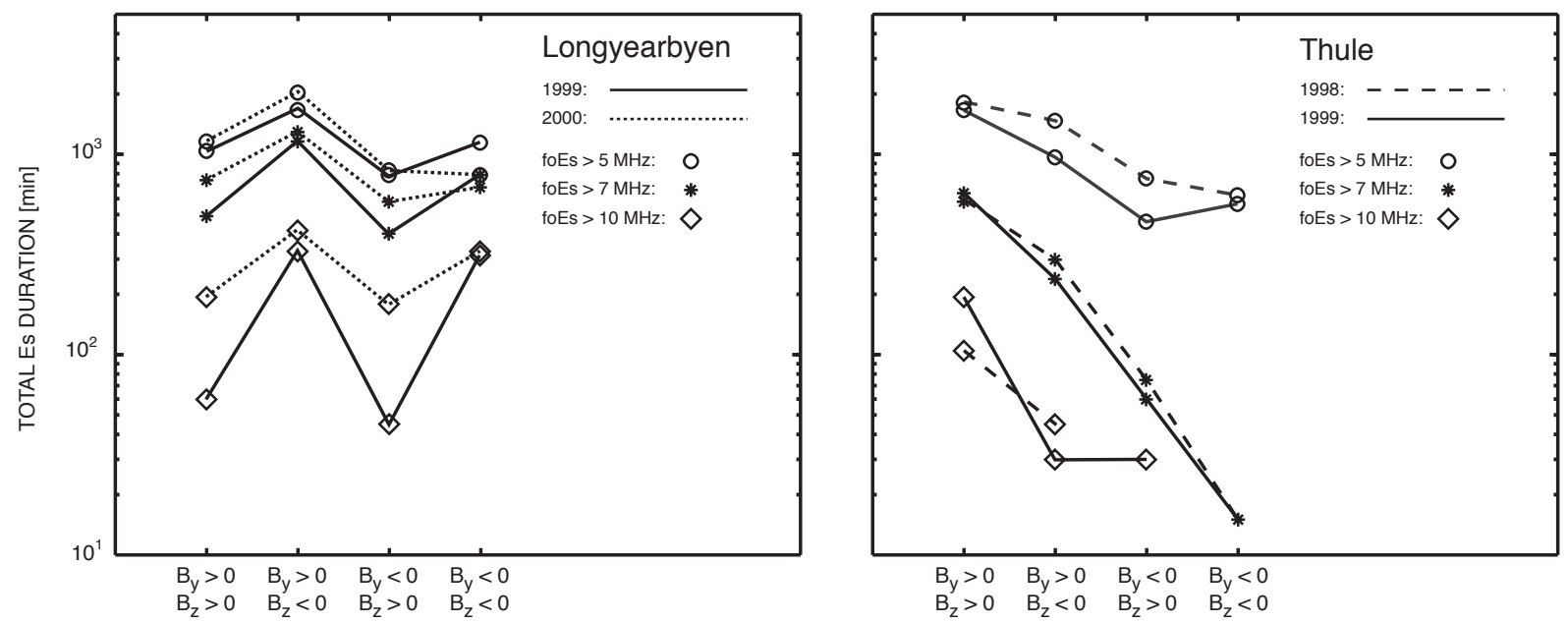

Fig. 5. The total time of Es occurrence at Longyearbyen and Thule when the IMF points in each of the four quardants of the $B_{y} B_{z}$ plane. Separate plots are made for each year and peak plasma frequencies higher than 5,7 and $10 \mathrm{MHz}$.

dense layers (panel $\mathrm{b}$ ) the dominance of positive $B_{y}$ is even more pronounced and the marginal $B_{z}$ distribution is also clearly shifted towards positive $B_{z}$. The IMF effect on the Es activity is clearer in the central polar cap than close to the border.

Further understanding of the Es dependence on the IMF comes from Fig. 4, where the total time of Es occurrence is depicted as a function of the IMF direction on the $y z$ plane. The full $360^{\circ}$-clock angle range is divided into $10^{\circ}$-bins and the total observational time of Es in minutes is shown within each bin for layers with peak plasma frequencies higher than $5,6,7$ and $10 \mathrm{MHz}$. The main features of the distributions are the same for all limits of the peak plasma frequency, indicating that both weak and strong layers are rather similarly affected by the IMF.

The behaviour of the distributions at Longyearbyen and Thule are quite different, as already indicated by Figs. 2 and 3. At Longyearbyen, layers are most often observed when the clock angle lies within the range $120^{\circ}-150^{\circ}$. This means positive $B_{y}$ and negative $B_{z}$, as already noticed in Fig. 2 . However, quite a few layers are also seen in the $80^{\circ}-90^{\circ}$ sector, when $B_{y}$ is positive and $B_{z}$ is zero or slightly positive. Another favourable sector is $240^{\circ}-290^{\circ}$, when $B_{y}$ is negative and $B_{z}$ is either positive or negative but smaller in comparison with $B_{y}$.

The role played by $B_{y}$ in the Es occurrence in the central polar cap is obvious when looking at Fig. 4, which shows that, at Thule the distributions are strongly oriented towards the positive $B_{y}$ axis. In particular, when the peak plasma frequency is high, only few layers are observed when $B_{y}$ is negative. Thus, the distributions greatly differ from those at Longyearbyen, where layers may often be produced even with negative $B_{y}$. At Thule, a maximum is observed at a clock angle of $80^{\circ}$, corresponding to positive $B_{y}$ and a small but positive $B_{z}$. Both distributions remain similar regardless of year and season (data not shown).

Figure 5 shows the total time of Es occurrence at Longyearbyen and Thule, when the IMF points in each of the four quardants of the IMF clock angle. The main observation from both sites is that a nearly identical pattern is repeated each year and it remains similar for all layers, regardless of their density. At Longyearbyen, the highest total occurrence rate of Es is observed when $B_{y}>0$ and $B_{z}<0$, the smallest one when $B_{y}<0$ and $B_{z}>0$, and nearly equal ones in the remaing two sectors. At Thule the occurrence rate is high when $B_{y}>0$ and small when $B_{y}<0$. When $B_{y}>0$, more layers are observed during positive than during negative $B_{z}$. So, layer occurrence is associated with positive $B_{y}$ at both of the stations. In addition, the Es layers occur more frequently during northward IMF at Thule and southward IMF at Longyearbyen.

\subsection{Diurnal variation of Es occurrence}

The diurnal variation of the Es occurrence at Longyearbyen is shown in Fig. 6 and and at Thule in Fig. 7. Four cases are considered according to the orientation of the IMF on the $y z$ plane: (a) $B_{y}>0$ and $B_{z}>0$, (b) $B_{y}>0$ and $B_{z}<0$, (c) $B_{y}<0$ and $B_{z}>0$, and (d) $B_{y}<0$ and $B_{z}<0$. In order to guarantee that the IMF stays within these sectors, data with $\left|B_{y}\right|$ or $\left|B_{z}\right|$ smaller than $2 \mathrm{nT}$ were not included in the analysis. The diurnal distributions are shown as a function of MLT, separately for each year and peak plasma frequencies higher than $5 \mathrm{MHz}, 7 \mathrm{MHz}$ and $10 \mathrm{MHz}$. Below the Es distributions, the magnitudes and directions of the calculated electric fields are shown. Figures 6 and 7 show that the main features are repeated every year at each site. Also, the distributions have more or less the same shape for dense and weak layers within the statistical limits set by the small number of high-density layers. 
a)
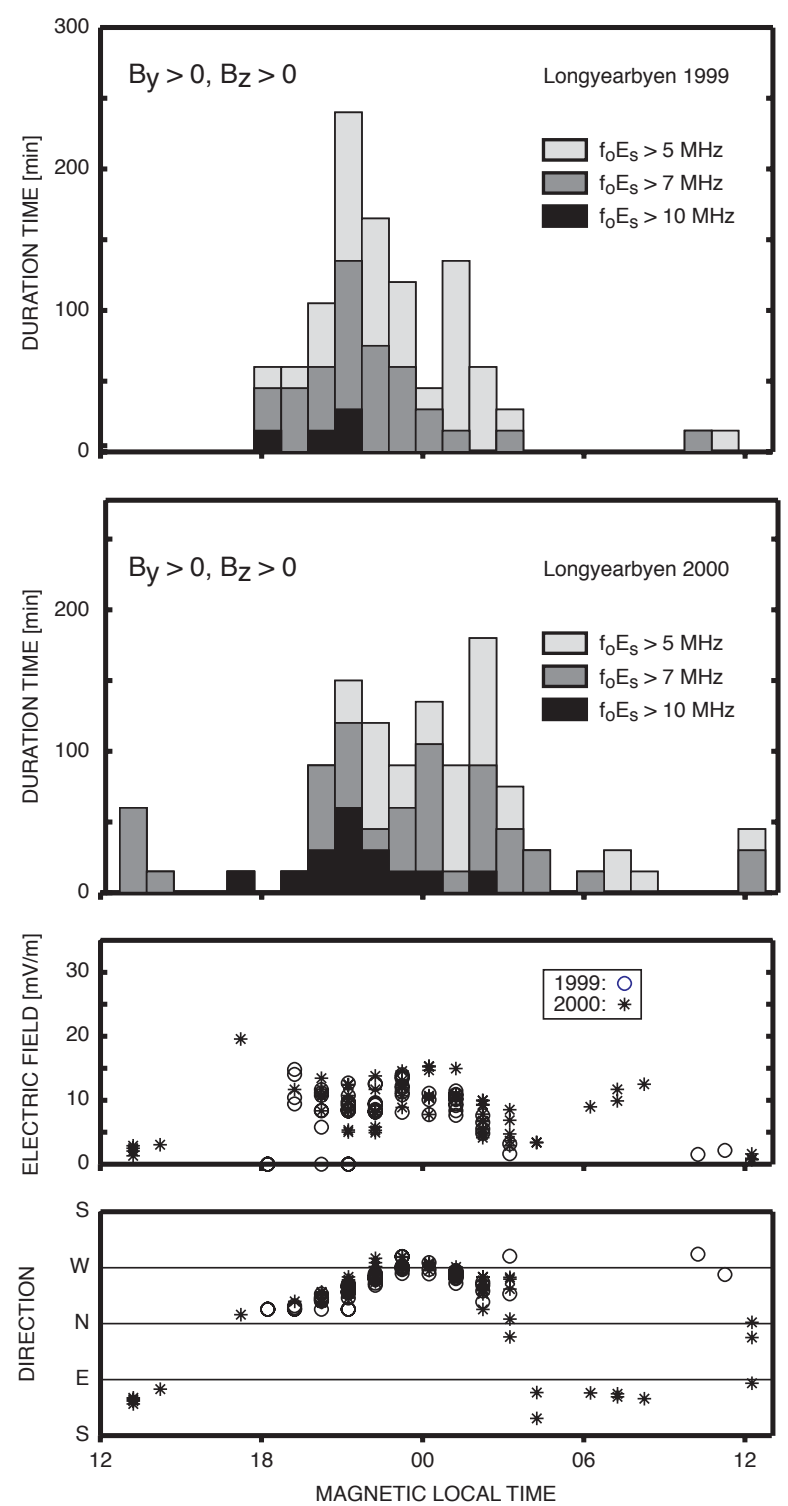

b)

$$
\mathrm{By}>0, \mathrm{~B}_{z}<0
$$
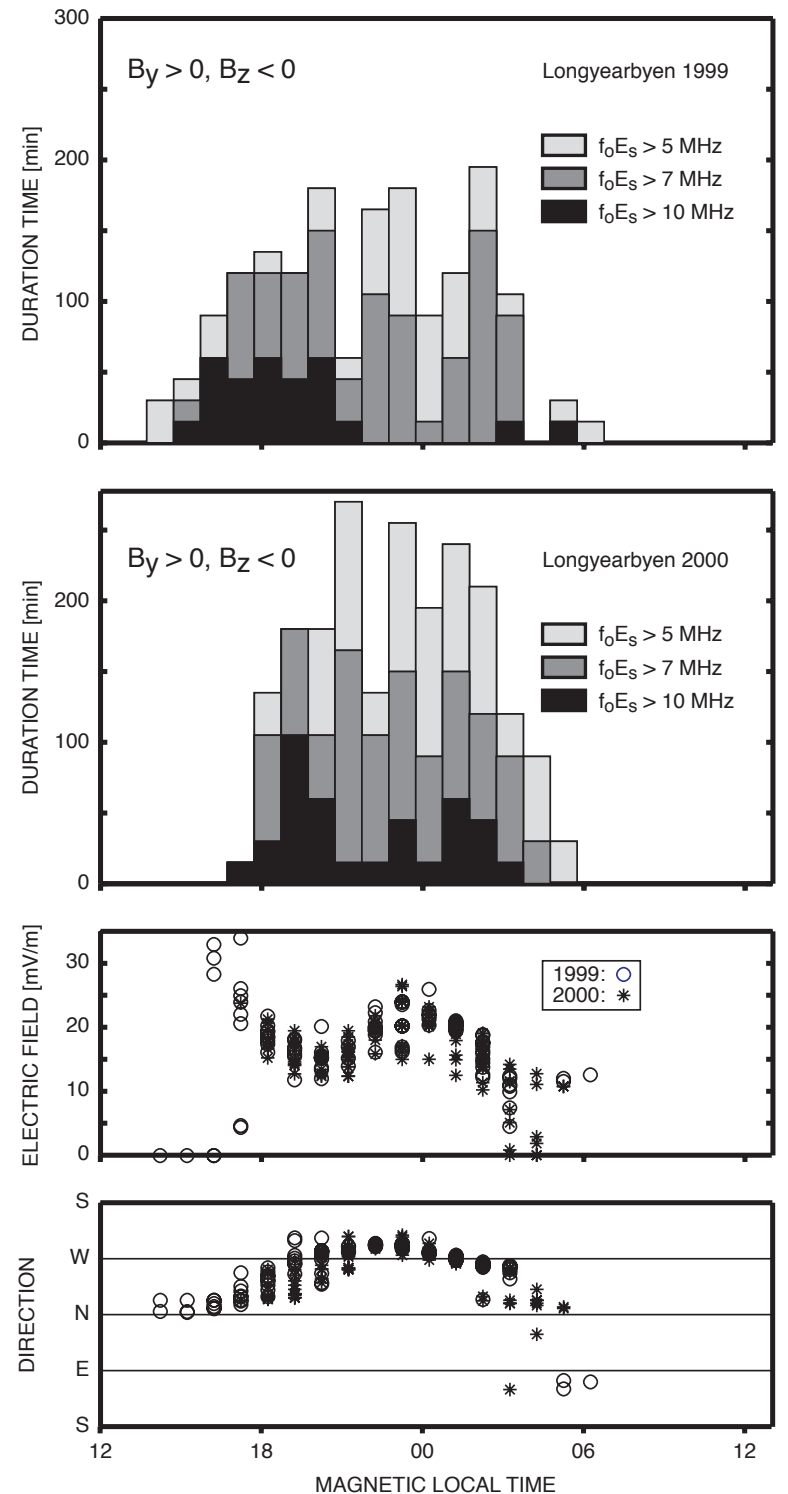

Fig. 6. (a, b) Diurnal distributions of Es occurrence in 1999 and 2000 at Longyearbyen, when (a) both $B_{y}$ and $B_{z}$ are positive (left) and (b) $B_{y}$ is positive and $B_{z}$ is negative (right). The amplitude and direction of the ionospheric electric field, calculated from simultaneous observations of the IMF using the statistical APL convection model are also shown. Cases with zero electric field come from incidents when the IMF is stronger than the highest value accepted by the APL model.

At Longyearbyen, the distributions are limited typically within the 18-06 MLT sector with hardly any daytime layers. The shapes of the distributions depend on the IMF direction. The broadest distributions, extending even to 15 MLT, occur for $B_{y}>0$ and $B_{z}<0$ (Fig. 6b), whereas the distribution for $B_{y}<0$ and $B_{z}<0$ contains a narrow, intense peak in the pre-midnight sector and a small, secondary maximum in the post-midnight sector (Fig. 6d). When $B_{z}>0$, the width of the distributions is almost independent of the sign of $B_{y}$, but the total time of layer occurrence is much smaller for $B_{y}<0$ (Fig. 6c) than for $B_{y}>0$ (Fig. 6a). All this indicates that at Longyearbyen, the IMF has a clear influence not only on the total abundance of the layers but also on the diurnal distribution of their occurrence probability.

Diurnal variations of Es layers have been observed at Troms $\varnothing$ by Kirkwood (1997) and at Sondrestrom by Bedey and Watkins (1998). Troms $\varnothing$ lies within the auroral oval and the position of Sondrestrom within the polar cap is 

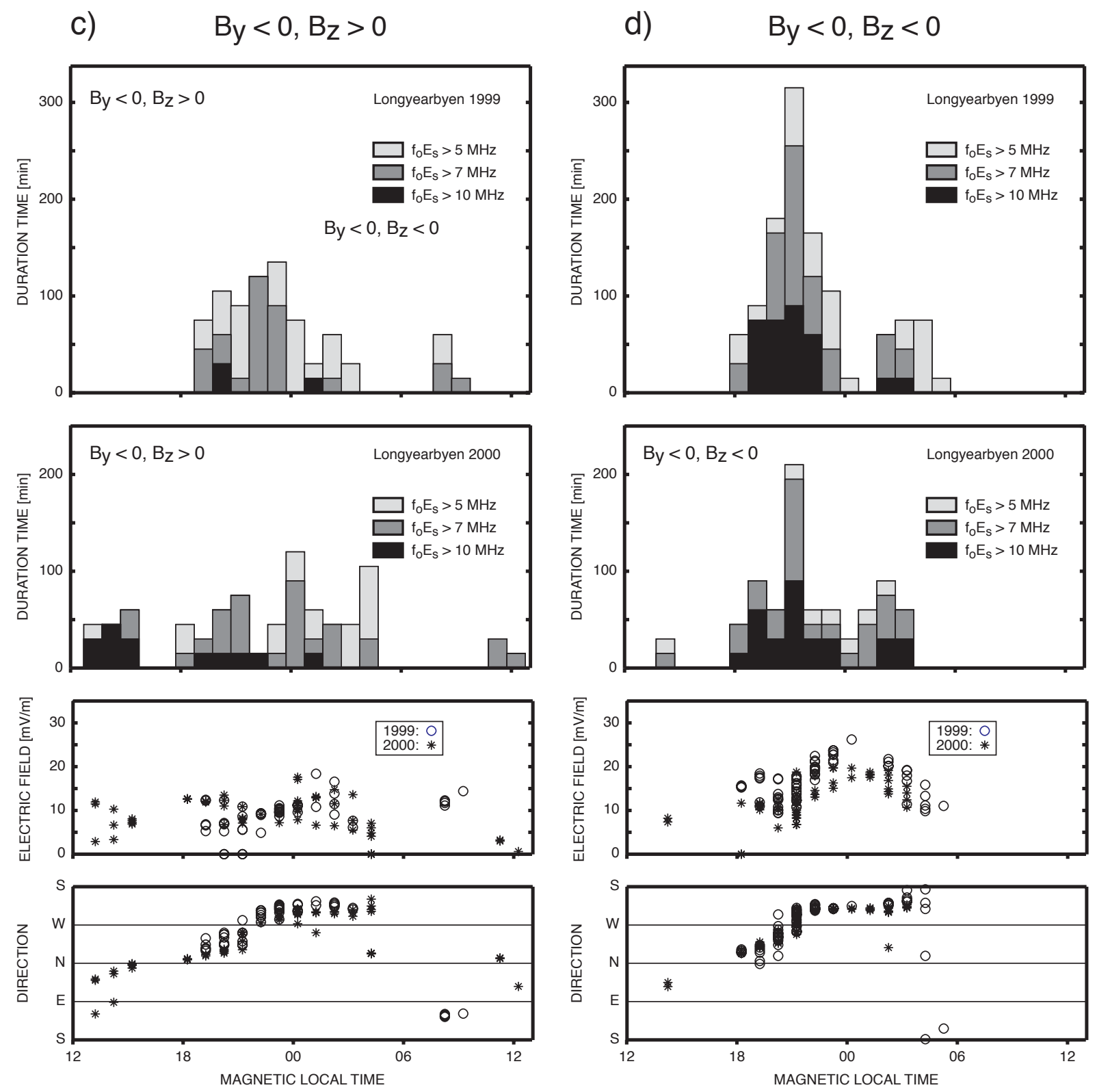

Fig. 6. Continued. Same as Fig. 6ab when (c) $B_{y}$ is negative and $B_{z}$ is positive and (d) both $B_{y}$ and $B_{z}$ are negative.

comparable to that of Svalbard. At Tromsø the Es layers are much more common between 19-03 MLT than at other times of the day. At Sondrestrom thin summer Es layers occur most likely at 20-23 MLT.

The diurnal variation at Thule is quite different from that at Longyearbyen. Layers are observed within a much wider time sector. The main feature for positive $B_{y}$ (Figs. 7a, b) is a minimum starting at about 00:00 MLT and extending to late morning. For negative $B_{y}$ (Figs. 7c, d), the total number of layers is small and, when the individual years are added together (see Paper II, Fig. 5), it is easier to see that dur- ing about 13-01 MLT the occurrence frequency of layers is about twice to that of 02-12 MLT. Hence, there is some indication that the occurrence minimum of Es starts a few hours earlier for positive $B_{y}$ than for negative $B_{y}$. Another aspect is that the shape of the diurnal variation of Es layers does not seem to depend on $B_{z}$. Generally, our results agree with Gabis (1999), who found that Es layers in the southern polar cap have a minimum between 00-12 MLT. This diurnal shape looks similar to what Wan et al. (1999) found at Casey $\left(-81^{\circ}\right.$ CGM Lat) in the Southern Hemisphere. The prenoon minimum is less apparent there, most likely because 
a)

$$
\mathrm{B}_{\mathrm{y}}>0, \mathrm{~B}_{\mathrm{z}}>0
$$
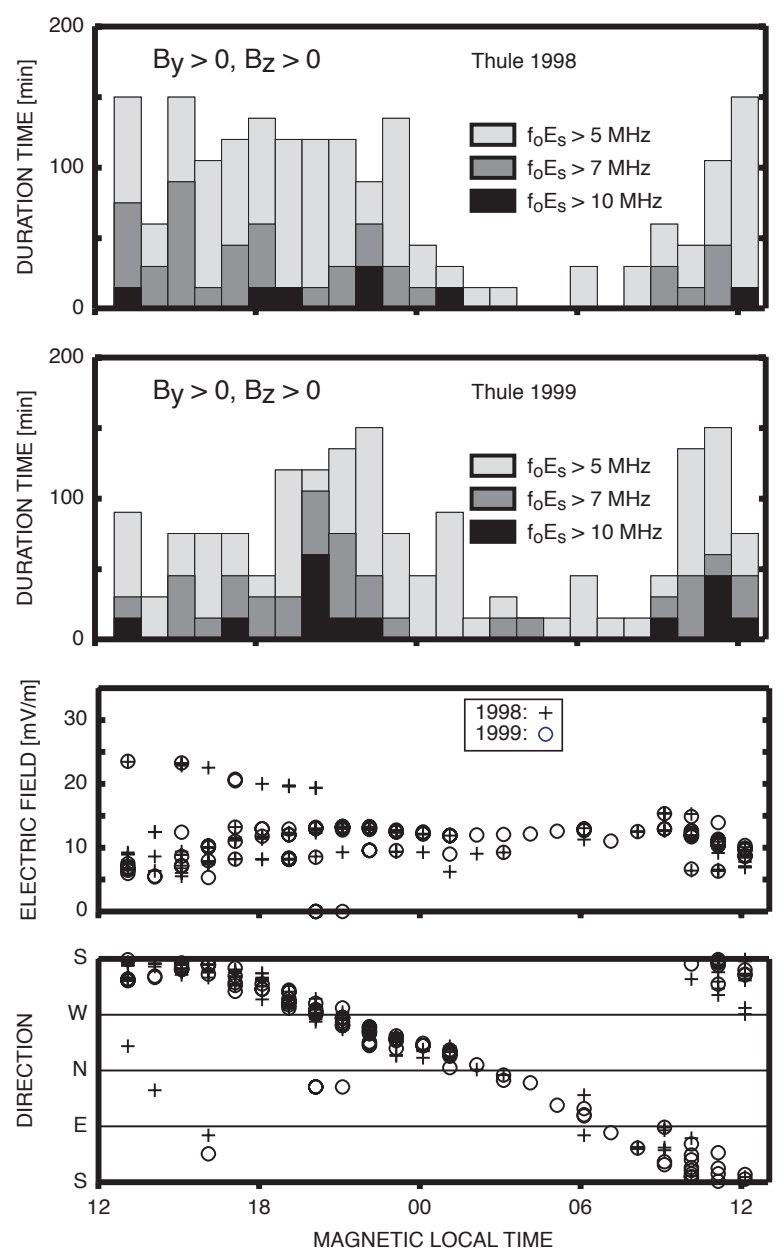

Fig. 7. (a, b) Same as Figs. 6a, b in 1998-1999 at Thule.

no limitation was imposed on the Es frequency. MacDougall et al. (2000a) found that, close to the northern geomagnetic pole, the peak occurrence of thin Es layers is around the local noon.

\section{IMF and electric field}

The plasma convection and electric field within the polar cap are mainly driven by the IMF, and the formation of Es layers at high latitudes is mainly explained by the electric field mechanism (Mathews, 1998; Kirkwood and Nilsson, 2000). Therefore, it is reasonable to assume that the electric field plays an important role in explaining the observed Es-IMF link. According to the electric field theory introduced by Nygrén et al. (1984), thin Es layers can be formed in the Northern Hemisphere when the electric field points in some direction between local geomagnetic north and west. The theory was extended by Bristow and Watkins (1991) by b) $\quad \mathrm{B}_{\mathrm{y}}>0, \mathrm{~B}_{\mathrm{z}}<0$
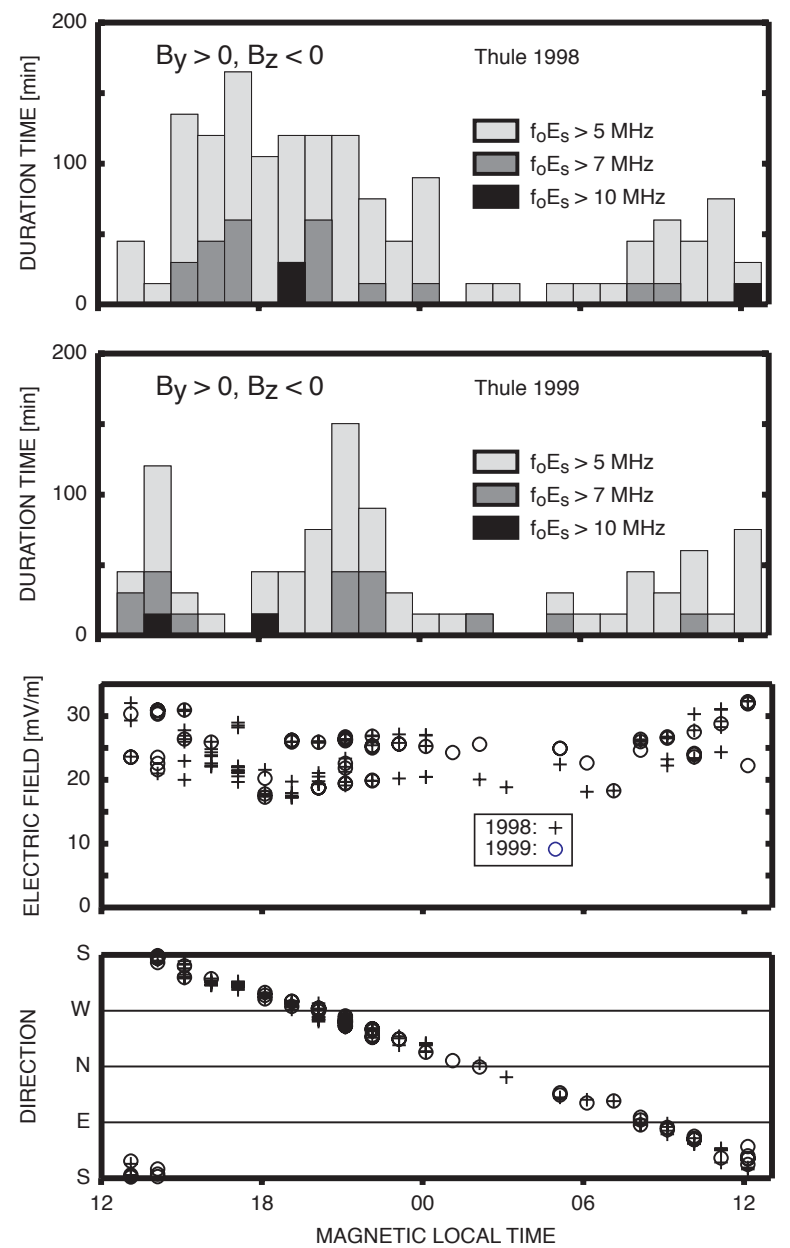

showing that Es could also form when the electric field direction lies between south and west. This result was confirmed both experimentally and theoretically by Kirkwood and von Zahn (1991), who also demonstrated that even an electric field direction pointing somewhat eastwards of south is capable of layer generation. The electric field strength, together with its direction is expected to affect the growth time, as well as the peak density of a layer. Hence, for a given electric field direction, the probability of observing a layer is expected to increase with increasing field strength. Concerning the field direction, a field pointing in the NW sector collects the available metallic ions quickly into a thin layer, while the layer grows more slowly and it will be thicker and weaker when the field points into the SW sector or between south and southeast (Kirkwood and von Zahn, 1991).

The electric field theory has been challenged by some experimental observations. Firstly, some Es layers have been observed during times when the electric field is not 

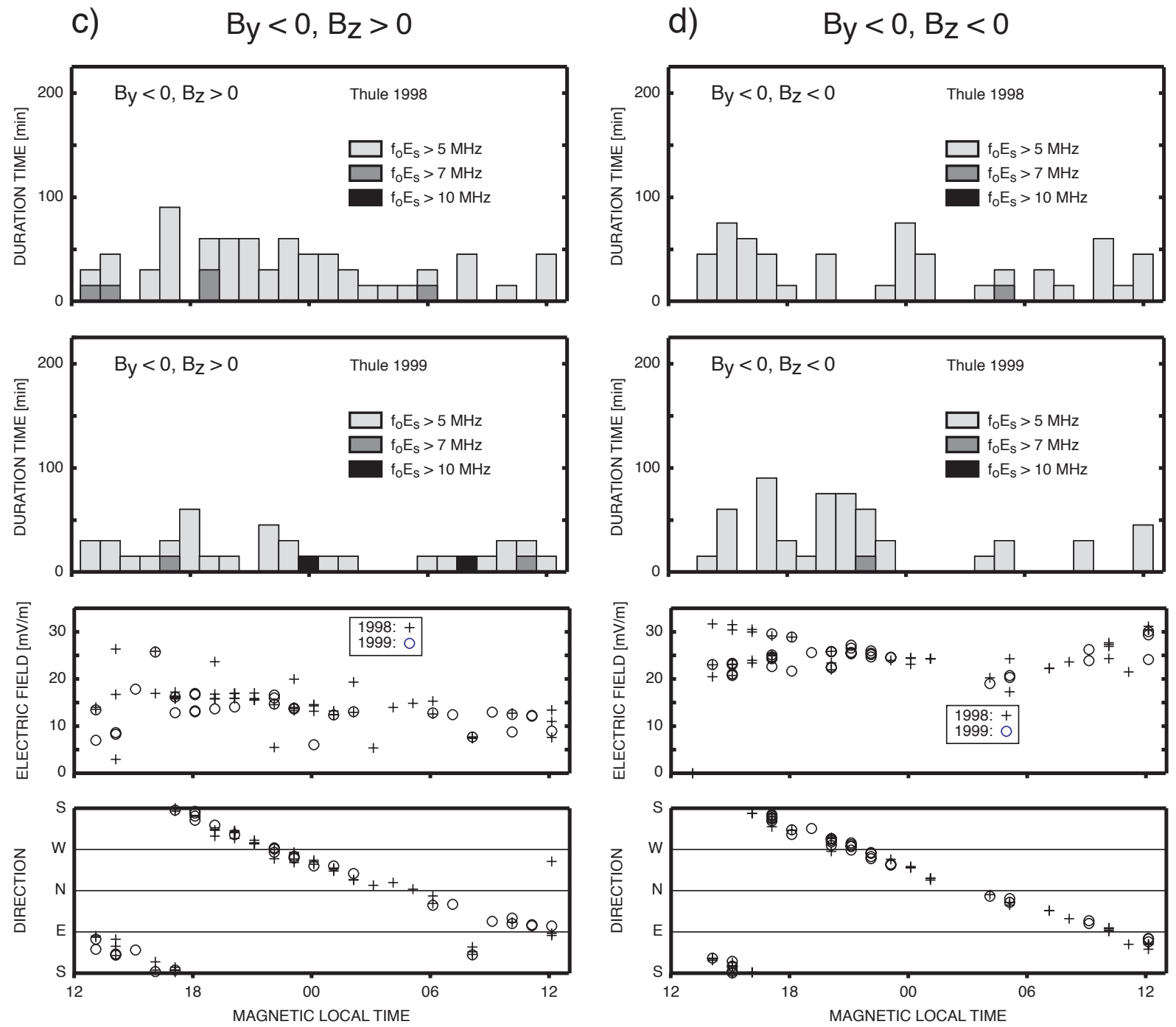

Fig. 7. Continued. (c, d) Same as Figs. 6c, d in 1998-1999 at Thule.

favourable (Bedey and Watkins, 2001; MacDougall et al., 2000a); the formation of such layers was theoretically attributed to the wind system (gravity waves). Secondly, appropriately directed electric fields do not guarantee the occurrence of Es layers (Bedey and Watkins, 1997), which opened the question of the abundance of metallic ions. It has been suggested that in these cases, Es layers could not form because metallic ions did not exist in sufficient amounts (Bedey and Watkins, 1998). In spite of these problems, observations of Es layers and electric fields overwhelmingly support the domination of the electric field in the Es formation at high latitudes (Bedey and Watkins, 1996; Bristow and Watkins, 1993; Kirkwood and von Zahn, 1991; Kirkwood, 1997; Parkinson et al., 1998). Consequently, we next try to explain some characteristics of the observed Es-IMF relationship by invoking the convection electric fields.

Concurrent measurements of the electric field were not available, and therefore we have utilised electric fields that were calculated from each IMF observation by means of the statistical APL convection model (Ruohoniemi and Greenwald, 1996). Since the true electric field can in reality considerably depart from the model value, agreement between the calculated electric field and layer occurrence is not expected in all individual cases. However, we can still investigate how well the calculated electric fields are consistent with the observed statistical distributions of Es occurrence. The two bottom panels below the Es distributions in Figs. 6 and 7 show the magnitude and direction of the calculated electric field at the times of observed Es layers. Each marker means an electric field from a 15-min interval of Es observation. A different marker is used for each year. 


\subsection{Electric field at Longyearbyen}

At Longyearbyen, layers are typically confined to 1806 MLT. Figure 6 indicates that most of the Es layers occur when the model predicts an electric field pointing in the $\mathrm{NW}$ or SW quadrant. This is consistent with the electric field mechanism. However, there are cases when the field direction clearly disagrees with the electric field theory. This happens, for example, at 12-15 MLT in 2001 (Fig. 6c), when intense layers are observed early in the afternoon, while the electric field is expected to lie in the NE sector.

A clear diurnal variation in the calculated electric field magnitude is seen at Longyearbyen. In addition, the magnitude also depends on the IMF direction. A combination of southward IMF and positive $B_{y}$ (Fig. $6 \mathrm{~b}$ ) produces the most intense electric field, on the average and, in the diurnal variation of the field strength, the main maximum is observed in the local afternoon and a secondary maximum at or just before midnight. When the field behaviour is compared with the Es distributions, one can see that the most intense layers occur early in the evening when the calculated electric field is intense and points in the NW-sector. In the pre-midnight sector, the occurrence of intense layers decreases while the electric field becomes weaker and turns closer to the west or even to the SW sector. Around midnight the occurrence of dense layers is still not high, regardless of the fact that the electric field magnitude reaches a secondary maximum and the direction is within the SW-sector. The layer occurrence is again enhanced later in the morning, although the field becomes weaker while turning back to the NW sector.

The second most intense electric field at Longyearbyen is obtained for a combination of southward IMF and negative $B_{y}$ (Fig. 6d). Again, the diurnal variation of the calculated electric field intensity shows a maximum at midnight, in a region of a SW-directed electric field, but this maximum corresponds to the Es occurrence minimum. The maximum of Es occurrence takes place around 20-21 MLT, when the electric field direction could be either in the NW or SW sector but when, according to the APL model, the magnitude of the electric field is lower than around midnight. The high occurrence of intense layers extends to earlier hours as far as 18 MLT. During this entire time interval the electric field direction lies in the NW-sector.

For northward IMF (Figs. 6a and c), the electric field magnitude is smaller, as expected, but a small maximum in the field magnitude around midnight remains. This, however, is not associated with a maximum in the Es occurrence.

The longest total cumulative time of layer occurrence in the four cases of Fig. 6 is found for $B_{y}>0$ and $B_{z}<0$ (Fig. 6b) and the shortest one for $B_{y}<0$ and $B_{z}>0$ (Fig. 6c). These IMF conditions correspond to the maximum and minimum average intensity of the calculated electric field, respectively. So, although the maxima in the MLT distributions of Es do not correspond to the maxima of electric field intensity, the integrated occurrence could be related to the average electric field magnitude.

\subsection{Electric field at Thule}

At Thule (see Fig. 7), the diurnal variation of the calculated electric field magnitude is small for any direction of the IMF. The electric field direction in the CGM-MLT coordinate system rotates through $360^{\circ}$ during the day. Layers occur from about noon until midnight, when the modelled electric field points in the SW and NW sectors, and, in some cases, also in the SE sector. The post-midnight-to-morning minimum coincides with times when the electric field is predicted to point in the NE sector, which is expected to prevent Es formation. The minimum is quite clearly visible when $B_{y}$ is positive (Figs. 7a and b). When $B_{y}$ is negative (Figs. $7 \mathrm{c}$ and d), this is not so obvious because the layers are rare and therefore the diurnal variation may be covered by statistical variation. We should notice that, although there is a minimum in the Es occurrence when the field points in the NE sector, layers still appear at these times. They could be produced by another mechanism associated with neutral wind or gravity waves (MacDougall et al., 2000b). As discussed above, it is also possible that the true electric field is actually different from the calculated field.

Also for Thule, the southward IMF ( $B_{z}$ negative) results in a more intense electric field than the northward IMF. However, this is not associated with more Es layers, as would be expected according to the electric field mechanism. It is rather the sign of $B_{y}$ which correlates with the layer occurrence. Clearly more layers are observed when $B_{y}$ is positive (Figs. 7a and b) than in the opposite case (Figs. $7 \mathrm{c}$ and d). We will discuss the possible explanations in the next section.

\section{Discussion}

Figures 2-7 prove that the occurrence of sporadic E within the polar cap is indeed closely connected to the IMF. The relationship between the Es occurrence and the IMF is different at the two sites. This is to be expected if we assume that the layers are mainly caused by the polar cap electric field. The two sites lie at different geomagnetic latitudes: Thule is located in the central polar cap at $85.4^{\circ} \mathrm{CGM}$ Lat, whereas Longyearbyen is located at a much lower latitude of $75.2^{\circ}$ CGM Lat. Therefore, the convection electric fields are expected to be different at these two sites for a given IMF direction.

Although a general agreement with the electric field theory is found, our observations contain features which cannot be explained solely by the calculated electric field direction and magnitude. These are as follows:

(1) The $B_{y}$ effect on the layer occurrence; $71 \%$ of the Es layers at Thule and $62 \%$ of the Es layers at Longyearbyen occur when $B_{y}>0$. 
(2) At Thule Es is slightly more abundant (56\%) during positive $B_{z}$, although the opposite would be expected, since negative $B_{z}$ produces a more intense electric field. Indeed, at Longyearbyen $61 \%$ of the total time of layer occurrence coincides with negative $B_{z}$.

(3) At Longyearbyen, for $B_{y}<0$ and $B_{z}<0$ (Fig. 6d), almost no layers appear at magnetic midnight.

(4) Layers are in some cases observed at times when the the modelled electric field points in the NE sector. Such layers are seen at both stations. Quite a few of them are observed at Longyearbyen, but at Thule, where layers are more rare, their relative occurrence is more significant.

Before trying to find some physical explanations for the items listed above, we will review the main observations of the IMF-Es relationship from earlier studies to show that items (1) and (2) are in accordance with earlier observations.

MacDougall et al. (2000a) studied data from three central northern polar cap stations. They found that height-spread Es layers were observed predominantly during $B_{y}$ positive and had no $B_{z}$ association, whereas thin Es layers were associated with both $B_{y}$ positive and $B_{z}$ positive. They argue that, due to the large inclination of the magnetic field, the electric field mechanism cannot be effective within the central polar cap. Instead, they explain the formation of polar cap Es by atmospheric gravity waves that move ionisation from the lower $\mathrm{F}$ region into the $\mathrm{E}$ region and compress the plasma at the reversals of vertical gravity wave motion (MacDougall et al., 2000b). It is doubtful, however, whether the $B_{y}$ effect can be entirely explained by this model. Furthermore, it will be shown in Paper II that, unlike that stated by MacDougall et al. (2000a), the electric field mechanism is effective within the central polar cap, even at Thule (85.4 ${ }^{\circ}$ CGM Lat).

Wan et al. (1999) studied Es in the southern polar cap, at a station whose geomagnetic latitude is approximately halfway between Thule and Longyearbyen. They found that negative $B_{y}$ correlates with layer occurrence, especially at midnight. Also, a $B_{z}$ effect was found, although it was weaker: positive $B_{z}$ correlated with Es in the evening sector. Simultaneous measurements of electric field by digisonde showed that negative $B_{y}$ was associated with an increase in the SW electric field (corresponds to the NW electric field in the Northern Hemisphere), and could thus qualitatively explain the $B_{y}$ dependence to some extent. Still, the $B_{z}$ effect could not be explained by the change in the electric field. They suggested that the layers could be a consequence of polar showers and squalls which occurred when $B_{z}$ was positive.

In this study, our aim was to investigate only thin layers and to reject height-spread and auroral type layers (see Sect. 2). Our Thule observations are in agreement with those by MacDougall et al. (2000a) in the central polar cap: Es occurs mostly during positive $B_{y}$. In particular, we find that intense layers are hardly ever observed when $B_{y}$ is negative. Positive $B_{y}$ correlates with layer occurrence also at Longyearbyen, but not so strongly as at Thule. Thus, both in the central polar cap and at lower polar cap latitudes in the Northern Hemisphere, Es formation is more likely during positive than negative $B_{y}$. According to Wan et al. (1999), a similar relation between Es layers and negative $B_{y}$ is found in the Southern Hemisphere. This agrees with our results, since positive $B_{y}$ in the Northern Hemisphere and negative $B_{y}$ in the Southern Hemisphere are associated with similar polar cap convection patterns (a more detailed discussion on the convection patterns is given below).

In the central polar cap, the $B_{z}$ effect is clearly weaker than that of $B_{y}$, and positive $B_{z}$ seems to correlate with Es generation. This is seen both in the present study and in the papers by Wan et al. (1999) and MacDougall et al. (2000a). The effect of positive $B_{z}$ seems to be similar in both hemispheres. Our results show that, close to the polar cap boundary, the role of $B_{z}$ is somewhat stronger and Es occurrence correlates with negative rather than positive $B_{z}$.

We find that the $B_{y}$ effect does not depend on magnetic local time. At Thule, positive $B_{y}$ increases the number of Es at all MLT sectors, where Es is expected to occur. A similar effect is also observed at Longyearbyen for all MLT sectors where Es is observed. There is one exception, however: an intense maximum of Es occurrence takes place during 2021 MLT when both $B_{z}$ and $B_{y}$ are negative. This peak is observed both in 1999 and 2000, so that it is hardly accidental. Therefore, we conclude that at Longyearbyen, there are actually more layers at 20-21 MLT when $B_{y}$ is negative than in the opposite case.

The important question is: What physical mechanism could account for the observed IMF effect? It would be most straightforward to try to explain the observations by the IMF control of convection. Then one immediately realises that $B_{z}$ would be expected to be the dominant factor since, during the southward IMF, the intensity of the convection electric field is highest both in the central polar cap, as well as close to its boundary (see Figs. 6 and 7). Indeed, at Longyearbyen negative $B_{z}$ correlates with Es, but at Thule there is a weak preference of Es layers to occur for positive $B_{z}$. However, the dominant factor at Thule turns out to be $B_{y}$ rather than $B_{z}$, and at Longyearbyen the $B_{y}$ effect, although smaller, is as strong as the $B_{z}$ effect.

In addition to the local electric field, the time history of plasma may also play a role in Es layer formation. The global plasma convection pattern is such that the dusk cell is larger in spatial extent than the dawn cell and its maximum potential is also greater (Ruohoniemi and Greenwald, 1996; Papitashvili and Rich, 2002). When $B_{y}$ is negative in the Northern Hemisphere, the zero potential line is aligned at about $00 \mathrm{MLT}$, and when $B_{y}$ is positive, the convection pattern and the zero line are rotated to an earlier time by a few hours. For positive $B_{y}$, the dusk cell in the Northern Hemisphere becomes more round and expands further towards dawn, and 
the centre of the dusk cell moves to an earlier time and to a higher latitude (Ruohoniemi and Greenwald, 1996; Papitashvili and Rich, 2002). In the Southern Hemisphere, similar effects are produced by negative $B_{y}$ and hence the convection patterns for positive $B_{y}$ in the Northern Hemisphere and negative $B_{y}$ in the Southern Hemisphere resemble each other (Papitashvili and Rich, 2002). Since a similar IMF asymmetry has been found in the Es occurrence (positive $B_{y}$ correlates with the Es occurrence in the Northern Hemisphere, negative $B_{y}$ in the Southern Hemisphere), the physical agent may be related to the IMF control of the convection pattern.

Next, we will discuss how the global convection pattern may be related to one important precondition of the layer formation: the availability of metallic ions. Bedey and Watkins (1998) note that the presence of a favourable electric field at Sondrestrom, Greenland, does not guarantee the existence of thin layers and they suggest that the reason is the variation in the ion abundance. Many Es layers consist of iron and magnesium (Huuskonen et al., 1988), which originate from meteor ablation in the E region. Neutral metallic atoms are ionised by collisions and also by charge exchange with $\mathrm{O}_{2}^{+}$ and $\mathrm{NO}^{+}$. Due to the slow recombination process, the lifetime of metallic ions is at least hours. Thus, during their lifetime, the ions can be transported long distances both horizontally and vertically. The horizontal transport in the E region is very slow, but if the ions are lifted up to the F region, they can be transported faster (Bedey and Watkins, 1997). Based on their model calculations, it was suggested by Bedey and Watkins (1997) that under the action of the electric field with an easterly component, metallic ions may be lifted to the $\mathrm{F}$ region in the dayside part of the polar cap. In the $F$ region the ions can be transported to the nightside, where they would precipitate, so that considerable horizontal variations in their abundance can be created in the E region. On the other hand, the calculations by Bedey and Watkins (1997) show that such regions are latitudinally narrow and don't extend close to the center of the polar cap. However, the convection pattern, and consequently the IMF, may play an important role in the spatial distribution of the metallic ions.

It has also been suggested that the availability of metallic ions would relate to particle precipitation within the polar cap (Wan et al., 1999). This may be possible, because the residue of meteor ablation is mainly neutral (see, e.g. Bedey and Watkins, 1998) and can be ionised by particle precipitation. Within the central polar cap the precipitation would be associated with polar auroral arcs, polar showers or squalls, which all appear during northward IMF (e.g. Kullen et al., 2002; Shinohara and Kokburn, 1996). Hence, production of metallic ions by particle precipitation and subsequent forming of Es should lead to a clear correlation of Es occurrence with positive $B_{z}$. Our observations, however, show only a weak correlation to positive $B_{z}$ within the central polar cap while the correlation to positive $B_{y}$ is strong and therefore local production of metallic ions can hardly be a dominant factor in explaining the results.
In conclusion, the spatial distribution of metallic ions may be related to their horizontal and/or vertical transport, which is controlled by the convective electric fields (Bedey and Watkins, 1997). Hence, the $B_{y}$ and $B_{z}$ effect, as explained in items (1) and (2), may originate from the IMF control on the availability of metallic ions via the global convection pattern. The layer formation may then occur as a result of the electric field mechanism, although neutral winds and atmospheric gravity waves may also play a role. A full investigation of these relations would imply extensive modelling, which is beyond the scope of this paper.

Problem (3), i.e. the observed minimum in Es occurrence around 00 MLT at Longyearbyen when $B_{z}<0$ and $B_{y}<0$, may tentatively be explained by substorm effects. Substorms tend to be associated with the southward IMF. Statistically, substorms start at $23 \pm 2$ MLT (e.g. Frey et al., 2004) and the effects may reach the latitudes of Longyearbyen. During substorm expansion, electric fields are typically highly variable. If electric fields are fluctuating rapidly both in direction and intensity, there may not be enough time for the layer to form (Wan et al., 2001). However, this suggestion remains open to further investigations.

Problem (4) calls for an explanation of the Es layers observed when the calculated electric field points in the NE sector. These (rather rare) cases can be due to more than one reason. Although the neutral wind is not usually dominant at high latitudes (Kirkwood, 1997; Kirkwood and Nilsson, 2000; Mathews, 1998), its role cannot be wholly neglected (Voiculescu and Ignat, 2005; Nygrén, 1990). Strong vertical winds or abrupt shears in the wind could occasionally dominate, leading to accumulation of ions into thin layers. Some of the layers could also be formed by gravity waves, as suggested by MacDougall et al. (2000b). It may also be possible that, as pointed out by Bedey and Watkins (2001), some of the Es layers were created elsewhere and transported into the observed area. Direct evidence of such horizontal drift of layers has been provided by Bedey et al. (2005). Finally, we should remember that the electric field is calculated from the statistical APL model (Ruohoniemi and Greenwald, 1996), and, after all, the true electric field direction may be within the favourable quadrants of the electric field theory in some of these cases.

\section{Conclusions}

The extended database covered by our study and the repetitive pattern seen for both stations, for all types of layers and for all years, allows us to conclude that the occurrence of Es layers within the polar cap is closely related to the interplanetary magnetic field. Since the polar cap electric field is controlled by the direction and magnitude of the IMF, it is a reasonable working hypothesis to assume that the electric field mechanism is the most important element in Es formation within the polar cap. In individual cases, other factors like 
neutral winds, gravity waves or polar cap precipitation phenomena may have an effect on layer generation.

Since measurements of the electric field were not available, we utilised the statistical APL model, which produces electric field vectors for given values of the IMF. This means that the calculated electric fields are valid only in a statistical sense and considerable disagreement may appear between the true electric field and the calculated one in individual cases (Ruohoniemi and Greenwald, 1996). Still, the observed MLT distributions of sporadic-E layers at Thule and Longyearbyen are mostly consistent with the calculated electric field pointing in a favourable direction, i.e. roughly in the NW or SW quadrant.

Close to the polar cap boundary, at Longyearbyen, more layers appear during negative than positive $B_{z}$. This is in accordance with the electric field mechanism, since during negative $B_{z}$ a more intense convection electric field is generated. However, we observed an additional IMF effect, which cannot directly be explained by the electric field theory. In the Northern Hemisphere, Es layers are correlated with positive $B_{y}$, both in the central polar cap and close to its boundary. The study by MacDougall et al. (2000a), based on data from central northern polar cap stations, also reveals a correlation between Es occurrence and positive $B_{y}$. Wan et al. (1999) find that in the Southern Hemisphere, negative $B_{y}$ correlates with Es formation. This is in accordance with our result because the effect of positive $B_{y}$ on convection in the Northern Hemisphere is equivalent to the effect of a negative $B_{y}$ in the Southern Hemisphere. Our results also show that the correlation between positive $B_{y}$ and Es is stronger in the central polar cap than close to its boundary. Our second observation, which cannot be directly explained by the electric field mechanism, is that during positive $B_{z}$, more Es layers are observed in the central polar cap than during negative $B_{z}$. Even this has been seen earlier both in the Northern and Southern Hemispheres (Wan et al., 1999, MacDougall et al., 2000a).

To summarise the results, we find that the Es observations are mostly in good agreement with the direction of the calculated electric field but not with the calculated electric field intensity. Therefore, some additional mechanism must be active. A key to the problem could be the fact that the availability of metallic ions is a necessary precondition for layer generation and this may be determined by horizontal motion. The electric field mechanism in its simple form only considers vertical ion motion. Bedey and Watkins (1997, 1998) have proposed that proper electric fields could lift the metallic ions to the F region, where they would be transported horizontally at long distances by the polar cap electric field. The metallic ions would then precipitate to $E$ region and could be compressed by the electric field to an Es layer in different parts of the polar cap. Obviously, the details of this mechanism depend on the convection pattern, which has an IMF dependence.

In conclusion, we suggest that the IMF plays a major role in the Es occurrence within the polar cap, since it controls the electric field which is capable of compressing the ions vertically into thin layers and since it may also control the background metallic ion density (via the horizontal transport and redistribution of ions). However, the three-dimensional ion motion within the polar cap should be modelled in order to verify this hypothesis.

Acknowledgements. We thank T. Hyart and I. Virtanen for their work on data collection and preliminary data analysis. The Longyearbyen ionosonde is run by the University of Leicester. World Data Center $\mathrm{C} 1$ is acknowledged for the Thule ionosonde data (station code THJ77). We acknowledge JHU/APL and P. Newel for providing the public use of the OVATION project. Grant (project No 51465) from the Academy of Finland and support from Space Institute at the University of Oulu is also gratefully acknowledged.

Topical Editor M. Pinnock thanks P.T. Jayachandran and another referee for their help in evaluating this paper.

\section{References}

Axford, W. I.: Note on a mechanism for the vertical transport of ionization in the ionosphere, Can. J. Phys., 39, 1393-1396, 1961.

Axford, W. I.: The Formation and Vertical Movement of Dense Ionized Layers in the Ionosphere Due to Neutral Wind Shears, J. Geophys. Res., 68, 769-779, 1963.

Bedey, D. F. and Watkins, B. J.: Seasonal occurrence of thin metallic ion layers at high latitudes, Geophys. Res. Lett., 23, 27892792, 1996.

Bedey, D. F. and Watkins, B. J.: Large-scale transport of metallic ions in the lower ionosphere, J. Geophys. Res., 102(A5), 96759681, 1997.

Bedey, D. F. and Watkins, B. J.: Diurnal occurrence of thin metallic ion layers in the high-latitude ionosphere, Geophys. Res. Lett., 25(20), 3767-3770, 1998.

Bedey, D. F., and Watkins, B. J.: Simultaneous observations of thin ion layers and the ionospheric electric field over Sondrestrom, J. Geophys. Res., 106(A5), 8169-8183, 2001.

Bedey, D. F., Watkins, B. J., Heinselman, C. J., Zellmann, R. L., and Jakes, E. M.: Observation of the horizontal drift of thin metallic ion layers over Sondrestrom, Geophys. Res. Lett., 32, L01102, doi:10.1029/2004GL021600, 2005.

Behnke, R. A. and Vickrey, J. F.: Radar evidence for $\mathrm{Fe}^{+}$in a sporadic-E layer, Radio Sci., 10, 325-327, 1975.

Bristow, W. A. and Watkins, B. J.: Numerical simulation of the formation of thin ionization layers at high latitudes, Geophys. Res. Lett., 18, 404-407, 1991.

Bristow, W. A. and Watkins, B. J.: Incoherent scatter observation of thin ionization layers at Sondrestrøm, J. Atmos. Terr. Phys., 55, 873-894, 1993.

Bristow, W. A. and Watkins, B. J.: Effect of the large scale convection electric field structure on the formation of thin ionization layers at high latitudes, J. Atmos. Terr. Phys., 56, 401-415, 1994.

Feldstein, Y. I. and Starkov, G. V.: Dynamics of auroral belt and polar geomagnetic disturbances, Planet. Space Sci., 15, 209-229, 1967.

Frey, H. U., Mende, S. B., Angelopoulos, V., and Donovan, E. F.: Substorm onset observations by IMAGE-FUV, J. Geophys. Res., A10304, doi:10.1029/2004JA010607, 2004. 
Gabis, I. P.: Anomalous ionisation of the ionospheric E region at the polar cap, Int. J. Geomagn. Aer. 1(2), 157-160, 1999.

Heppner, J. P. and Maynard, N. C.: Empirical high-latitude electric field models, J. Geophys. Res., 92, 4467-4489, 1987.

Huuskonen, A., Nygrén, T., Jalonen, L., Bjørnå, N., Hansen, T. L., Brekke, A., and Turunen, T.: Ion composition in sporadic E layers measured by the EISCAT UHF radar J. Geophys. Res., 93, 14 603-14610, 1988.

Kirkwood, S.: Thin ion layers in the high-latitude ionosphere, Adv. Space Res., 19, (1)149-(1)158, 1997.

Kirkwood, S. and von Zahn, U.: The role of magnetospheric electric fields in producing auroral zone thin layers, Adv. Space Res., 12(10), 225-228, 1991.

Kirkwood, S. and Nilsson, H.: High-latitude sporadic-E and other thin layers - the role of magnetospheric electric fields, Space Sci. Rev., 91, 579-613, 2000.

Kullen, A., Brittnacher, M., Cumnock, J. A., and Blomberg, L. G.: Solar wind dependence of the occurrence and motion of polar auroral arcs: A statistical study, J. Geophys. Res., 107, 1362, doi:10.1029/2002JA009245, 2002.

MacDougall, J. W., Jayachandran, P. T., and Plane, J. M. C.: Polar cap sporadic E: part 1, Observations, J. Atmos. Solar-Terr. Phys., 62, 1155-1167, 2000a.

MacDougall, J. W., Plane, J. M. C., and Jayachandran, P. T.: Polar cap sporadic E: part 2, Modeling, J. Atmos. Solar-Terr. Phys., 62, 1169-1176, 2000b.

Mathews, J. D.: Sporadic E: current views and recent progress, J. Atmos. Solar-Terr. Phys., 60, 413-435, 1998.

Nygrén, T., Jalonen, L., Oksman, J., and Turunen, T.: The role of electric field and neutral wind direction in the formation of sporadic E layers, J. Atmos. Terr. Phys., 46, 373-381, 1984.

Nygrén, T.: Vertical plasma transport due to electric fields and neutral winds in the auroral ionosphere, Adv. Space Res., 10 (6), 297-305, 1990.

Nygrén, T., Aikio, A. T., Voiculescu, M., and Ruohoniemi, J. M.: IMF effect on sporadic E at two polar cap sites: Part II - Electric field, Ann. Geophys., 24, 901-913, 2006.
Papitashvili, V. O., Belov, B. A., Faermark, D. S., Feldstein, Y. I., Golyshev, S. A., Gromova, L. I., and Levitin, V. E.: Electric potential patterns in the northern and southern polar regions parametrized by the interplanetary magnetic field, J. Geophys. Res., 99, 12 251-12 262, 1994.

Papitashvili, V. O. and Rich, F. J.: High-latitude ionospheric convection models derived from Defense Meteorological Satellite Program ion drift observations and parameterized by the interplanetary magnetic field strength and direction, J. Geophys. Res., 107, doi:10.1029/2001JA000264, 2002.

Parkinson, M. L., Dyson, P. L., Monselesan, D. P., and Morris, R. J.: On the role of electric field direction in the formation of sporadic E-layers in the southern polar cap ionosphere, J. Atmos. and Solar-Terr. Phys., 60, 471-491, 1998.

Rich, F. J. and Hairston, M.: Large-scale convection patterns observed by DMSP, J. Geophys. Res., 99, 3827-3844, 1994.

Ruohoniemi, M. and Greenwald, R. A.: Statistical patterns of highlatitude convection obtained from Goose Bay HF radar observations, J. Geophys. Res., 101, 21 743-21 763, 1996.

Shinohara, I. and Kokburn, S.: Statistical propersties of particle precipitation in the polar cap during intervals of northward interplanetary magnetic field, J. Geophys. Res., 101, 69-82, 1996.

Wan, W., Parkinson, M. L., Dyson, P. L., Breed, A. M., and Morris, R. J.: A statistical study of the interplanetary magnetic field control of sporadic E layer occurrence in the southern polar cap ionosphere, J. Atmos. Solar-Terr. Phys., 61, 1357-1366, 1999.

Wan, W., Liu, L., Parkinson, M. L., Liu, R., He, L., Breed, A. M., Dyson P. L., and Morris, R. J.: The effect of fluctuating ionospheric electric fields on Es-occurrence at cusp and polar cap latitudes, Adv. Space Res., 27, 1283-1288, 2001.

Weimer, D. R.: Models of high-latitude electric potentials derived with a least error fit of spherical harmonic coefficients, J. Geophys. Res., 100, 19595-19607, 1995.

Whitehead, J. D.: The formation of sporadic E layer in the temperate zones, J. Atmos. Terr. Phys., 20, 49-58, 1961.

Whitehead, J. D.: Production and prediction of sporadic E, Rev. Geophys. Space Phys., 8, 65-144, 1970. 